\title{
Laser - Multiphased Applications in Dentistry
}

\author{
Alexander Asokan ${ }^{1, *}$, Bharath KP ${ }^{2}$, Halasawmy V. kambalimath ${ }^{1}$, Raju U. Patil ${ }^{1}$ \\ ${ }^{1}$ Department of Pedodontics and preventive dentistry, Rishiraj College of dental sciences and research Centre, Bhopal, India \\ ${ }^{2}$ Department of Pedodontics and preventive dentistry, Bapuji College of dental sciences, Davengree, India \\ *Corresponding author: alexlites@gmail.com
}

Received October 17, 2014; Revised October 28, 2014; Accepted December 18, 2014

\begin{abstract}
This article reviews about the nature of lasers and their mechanism in diagnosing and treating various dental diseases and reality behind its usage and procedure with the objective of compiling key factors in achieving clinical modalities of laser therapy in dentistry. It also discusses the laser -dental tissue interactions for better understanding.
\end{abstract}

Keywords: laser, applications, pulp dentin complex

Cite This Article: Alexander Asokan, Bharath KP, Halasawmy V. kambalimath, and Raju U. Patil, "Laser Multiphased Applications in Dentistry." International Journal of Dental Sciences and Research, vol. 2, no. 6B (2014): 26-30. doi: 10.12691/ijdsr-2-6B-8.

\section{Introduction}

The earliest use of photo chemotherapy, or the use of an exogenous photosensitizer to absorb light and render a therapeutic effect, dates back to 1400B.C. Indians used a herbal drug called 'psoralen' to treat vitiligo. [1] Lasers have benefited from decades of research and have their basis in certain theories of quantum mechanics, formulated during the early 1900's by physicist Bohr. The first lasers to be marketed for intra-oral use generally were $\mathrm{CO}_{2}$ lasers with otorhinolaryngologic clearances authorized by the FDA. During the 1970's and 1980's, intra-oral use of $\mathrm{CO}_{2}$ lasers was confined primarily to specialists, such as ENT surgeons, oral surgeons, and some periodontists. [2] The fact that Dr Terry Myers and his brother, William, an ophthalmologist, selected the $\mathrm{Nd}$ :YAG laser for their experiments on removal of incipient caries should come as net surprise due to the widespread use of this type of laser in ophthalmology by the mid 1980's (Myers and Myers, 1985) [1].

\section{Caries Detection and Pulpal Diagnosis Using Laser Light}

In 1980's, a technique focusing on the natural green fluorescence of tooth tissue was developed. The technique used a $488 \mathrm{~nm}$ argon laser to discriminate bright green fluorescing healthy tooth tissue from poorly fluorescing caries lesions. The technique was developed further in the early 1990s, into what is now known as Quantitative Light-induced Fluorescence (QLF). [3] In the quantitative light-induced fluorescence (QLF) method, yellow fluorescence is induced by irradiating the tooth by visible light in the blue-green region. More recently, laser light was used to induce fluorescence of enamel in a sensitive, non-destructive method for detection of enamel demineralization and dental caries. [4] A filter is used to eliminate the excitation wavelengths from the emitted light so that only the fluorescent is detected. The lesions appear as shadow images against the bright fluorescent background of the sound enamel. It is not known at this time what causes this fluorescence and why the carious lesion does not. The shadow may be primarily due to increased scattering in the lesion. The image can be stored, measured, and quantified in terms of shape and area. Images at subsequent times can be taken similarly, and by subtraction the clinician can decide on lesion reversal or progression. The technique is in use for clinical trials in several sites. The reproducibility of the method is high and shows great promise for the limited types of lesions that can be detected by it. There have been problems of stain confounding the signal. It remains to be seen whether this technique will be useful as a general clinical tool for the practicing pediatric and general dentist [5].

QLF uses the intrinsic fluorescence of the teeth within the yellow-green spectrum of visible light. The tooth is illuminated with a blue light that is emitted from a handpiece and causes the tooth structure to fluoresce and the image is captured with a colormicrovideo chargecoupled device camera. The data are then stored and analyzed by image analysis computer software. The tooth is seen on the computer monitor as fluorescent green; dark areas indicate mineral loss. With the computer software, the image can be saved and compared over time to track demineralization or remineralization. The fluorescence of the dental tissue has a direct relation with the mineral content of the enamel. Sometimes a red fluorescence appears, which has an extrinsic source and is emitted by porphyrins metabolized by bacteria in dental biofilm, calculus, or infected carious lesions usually indicate a high caries activity. QLF is a useful method for visualizing dental biofilm that is not visible clinically under ordinary lights. Red fluorescence from bacterial origin as observed 
in calculus, plaque and (advanced) caries, allows insight into oral hygiene levels, visualization of leaking margins of sealants and restorations and is furthermore suggested for use during caries excavation in children. The area of concern can be tracked over time to evaluate the success of remineralization efforts. QLF has a limited ability to detect interproximal carious lesions that involve two tooth surfaces, as the optical axis of the QLF has to be oriented perpendicular to the tooth surface and the amount of dehydration of a tooth surface can alter the intensity of the fluorescence At the present time, QLF is being used for research purposes more than for clinical practice. In the very near future, the cost of QLF should be lowered to make it more widely available for private practices [6,7].

Krause F compared the conventional laser fluorescence system (Diagnodent) and a novel laser fluorescence pen device (Diagnodent pen) for the detection of occlusal caries and they concluded that the new laser fluorescence device seems to be suitable for occlusal caries diagnosis. However, proposed guidelines for the clinical use of laser fluorescence readings of the conventional fluorescence device cannot be transferred to the Diagnodent pen. When performing measurements with the new pen device for caries diagnosis, lower values have to be expected than for the conventional system [8].

Laser Doppler Flowmetry: Laser Doppler Flowmetry (LDF) is a noninvasive, objective; painless, semiquantitative method of recording pulpal blood flow. [9] Morikawa et al. in 1972 developed the Laser Doppler Flowmetry to assess blood flow in microvascular systems such as the retina, the renal cortex or the skin. Gazelius et al. adopted this original method to monitor blood flow in intact teeth. The original technique used a light beam from a He-Ne laser emitting at $632.8 \mathrm{~nm}$. Other wavelengths of semi-conductor laser have also been used: $780 \mathrm{~nm}$ and $780-820 \mathrm{~nm}$ at low power of 1 to $2 \mathrm{~mW}$. [10] Laser Doppler flowmetry is an optical measuring method that enables the number and velocity of particles conveyed by a fluid flow to be measured. The particles must be big enough to scatter sufficient light for signal detection but small enough to follow the flow. [9] Laser light is transmitted to the dental pulp by means of a fibre optic probe placed against the tooth surface. Two equalintensity beams (split from a single beam) intersect across the target area. The scattered light beams from moving red blood cells will be frequency-shifted whilst those from the static tissue remain unshifted in frequency. The reflected light, composed of Doppler-shifted (light reflected by a moving object is Doppler-shifted) and unshifted light, is returned by an afferent fibre within the same probe to photodetectors in the flowmeter and a signal is produced. The photo-detectors convert the interference pattern arising from the mixing of shifted and unshifted light into a semiquantitative measurement of blood flow, termed the Flux signal, which is measured in arbitrary units. The received signal is calculated with a preset algorithm in the LDF machine. The LDF output signal or Flux can be simplified as a function of the product of red blood cells concentration as well as their mean velocity. In fact, flux is the number of moving red blood cells per second times their mean velocities. When used to assess the vitality of teeth, the size of the Flux signal obtained from a healthy vital control tooth can be compared with that of the suspected non-vital tooth. The Flux signal from a tooth with a vital pulp should be greater than from a tooth with a non-vital pulp [11].

It should be emphasized that the optical properties of a tooth change when the pulp becomes necrotic and this can produce changes in the LDF signal that are not due to differences in blood flow. Moving red blood cells causes the frequency of the laser beam to be Doppler shifted and some of the light to be backscattered out of the teeth. The reflected light is detected by the photocell on the tooth surface and its output is proportional to the number and velocity of the blood cells. The design of probes, probe holder characteristics, gingival isolation devices, wavelengths, bandwidths, laser powers etc. have been found to influence the sensitivity and specificity of LDF. It is difficult to obtain the laser reflection from some teeth. The anterior teeth, in which the enamel and dentin are thin, generally do not present a problem. In the molar teeth, the enamel and dentin are thick however. The advantage of this diagnostic method is that it allows painless diagnosis. The laser Doppler flowmetry method is useful in detection of pulp vitality of immature or traumatized teeth and for patients who are sensitive to tooth pain. [13] Odor T.M. investigated the effect of wavelength and bandwidth on laser Doppler flow meter signals from vital and root-filled teeth, and to establish their sensitivity and specificity. The $810-\mathrm{nm} / 3.1 \mathrm{kHz}$ combinations offered the greatest sensitivity and specificity as a test to distinguish between root-filled and vital teeth. This combination was best. [12] Sasano.T determined whether use of transmitted laser light would enable a better assessment of human pulpal vitality than back-scattered light does (LDF: laser Doppler flowmetry). The results indicated that transmitted laser light would be useful for the assessment of tooth pulp vitality because the blood flow signals did not include flow of non-pulpal origin, and because its output signals and response to blood flow changes were clear and could easily be monitored. [14] Chaiyavej.S. investigated the response of intradental A- and C-fibers during tooth cutting by Er: YAG laser. They concluded that during the tooth cutting, Er: YAG laser was more effective in activating intradental A-fibers compared with micro motor and caused the activation of intradental C-fibers. [15] The hot gutta-percha method is commonly applied for the differential diagnosis of vital versus non-vital dental pulp. This method has a disadvantage in that pain response cannot always be obtained because of the thick enamel and dentin or the high pain perception threshold of the dental pulp. The laser stimulation method by pulsed $\mathrm{Nd}$ : YAG laser was reported as an alternative to the hot guttapercha method. The pain induced by pulsed Nd:YAG laser was reported to be mild and tolerable compared with the pain induced by the conventional electric pulp tester [13].

\section{Laser Cavity Preparation and Restoration}

Since the Excimerlaser showed only a restricted ability in clinical use, mainly because of ablation and technical reasons, the erbium based laser systems (Er:YAG and Er,Cr:YSGG) have become standardized treatment tools for dental hard tissues in paediatric restorative dentistry as well. Through their specific ablation mechanism, they cause a micro-retentive pattern in the walls of the prepared 
cavity. [16] The Er:YAG, Er:YSGG and Er,Cr:YSGG lasers operate at wavelengths of 2940, 2790, and 2780nm, respectively. These wavelengths correspond to the peak absorption range of water in the infrared spectrum, although the absorption of the Er:YAG laser $(13,000 \mathrm{~cm}-1)$ is much higher than that of the Er:YSGG $(7000 \mathrm{~cm}-1)$ and Er,Cr:YSGG (4000cm-1). Since all three lasers rely on water-based absorption for cutting enamel and dentine, the efficiency of ablation is greatest for the Er:YAG laser. These laser systems can be used for effective caries removal and cavity preparation without significant thermal effects, collateral damage to tooth structure, or patient discomfort especially required among children. Normal dental enamel contains sufficient water (approximately 12 per cent by volume) that a water mist spray coupled to an Er-based laser system can achieve effective ablation at temperatures well below the melting and vaporization temperatures of enamel. Er-based dental lasers can also be used to remove composite resin and glass-ionomer cement restorations, and to etch tooth structure. [17,18] The strong absorption property of water is used to hold down the development of heat during ablation: since water absorbs the laser radiation better than the dental hard tissue, the temperature rise of the tissue is relatively low during ablation. The water reaches its boiling point and causes a microexplosion in the tooth (spallation). This divides the surrounding tissue into very small pieces and at the same time blows them apart. Since the explosion occurs in water, this method is called "water induced ablation". The pulse energy should be as small as possible to prevent changes to the structure of the tissue. Since some conduction of heat cannot be avoided, even though most of the radiation is absorbed by the water, a water spray has to be used for cooling [16].

Ablation crater formed by Er:YAG laser shows roughness of the cavity ground and uneven ablation edge. In enamel, roughness of the cavity is easily visible, but in dentin the shard like character is missing. The ablation is less irregular than in enamel because dentin is less brittle. The peritubular dentin is harder and therefore the intertubular dentin is ablated more extensively. The dentin tubules thus appear to protrude into the preparation area like chimneys. Since the mode of action for Er:YAG and Er,Cr:YSGG is same. The observed effects are similar. With the enlargement of the diameter of laser tip, the intensity on the surface decreases, since the pulse energy spreads to a bigger surface. Thus smaller tip results in deeper and retentive pattern whereas larger tip diameter causes shallower and more even pattern, which corresponds to the decreasing intensity. ${ }^{16}$ Powell concluded that the energy densities generated during laser curing did not affect the pulp or the surrounding enamel. [19] Anic measured a temperature increase of only $2.50 \mathrm{C}$ while laser curing of Class I restorations in vitro. The highest surface temperature of the cured resins was $13.80 \mathrm{C}$, which was due to exothermic polymerization reaction and light energy. [18] Westerman investigated artificial caries formation on the surface of a root. They came to the same conclusion of reduced caries progression after laser polymerization. They found an overall up to only $65 \mu \mathrm{m}$ with laser curing. These results show a significant increase resistance to demineralization and caries formation in cementum and dentin due to argon laser irradiation [21].
Prevention of dentin fractures:Lin C.Pattempted to use lasers to fuse a low melting-point bioactive glass to fractured dentin. This report is focused on the phase, compositional, and morphological changes observed by means of X-ray diffractometer, Fourier transforming infrared spectroscopy, and SEM energy depressive X-ray spectroscopy in human dentin after exposure to $\mathrm{Nd}$ : YAG laser. The irradiation energies were from $150 \mathrm{~mJ} /$ pulse-10 pps-4 s to $150 \mathrm{~mJ} /$ pulse-30 pps-4 s. Based on the results the irradiation energy of $\mathrm{Nd}$ : YAG laser used to fuse a low melting-point bioactive glass to dentin is $150 \mathrm{~mJ} /$ pulse- 10 pps-4s pps points per second) [22].

\section{Pulp Treatment by Lasers}

To date, the types of lasers used in pulp treatment procedures are: CO2, Nd:YAG, Er:YAG, Er,Cr:YSGG, 980nm GaAlAs diode laser and 810nm GaAlAs diode laser. [23] Treatment of indirect pulp capping in children could be performed by laser. The discovery of closure of dentinal tubules by laser energy and the sedative effects on pulpitis has led to the development of several new treatments that are soon to be put into practice. Deep cavities and hypersensitive cavities that require sedative treatment are some of the indications for laser treatment [13]. Areduction in the dentin permeability achieved by sealing the dentinal tubules is of paramount importance. Nd:YAG and $9.6 \mu \mathrm{m} \mathrm{CO} 2$ lasers can be used for this purposes. [24] When using the pulsed Nd:YAG laser, it is necessary to combine the application of black ink on the tooth surface and air spray cooling to prevent dental pulp damage resulting from the laser energy provided by $2 \mathrm{~W}$ and $20 \mathrm{pps}$ for less than 1 second on the area. Various clinical cases treated using this method produced no postoperative pain. The mechanism of this treatment has been proved by various studies, which described the degree of dye penetration has decreased after laser irradiation of the dentin surface. When using the $9.6 \mu \mathrm{m}$ $\mathrm{CO} 2$ laser, the dental tissue must not be irritated by exposure to high-energy lasers for long periods of time. The $9.6 \mu \mathrm{m} \mathrm{CO} 2$ laser energy is well absorbed by the hydroxyapitite of enamel and dentin, causing tissue ablation, melting and resolidification. In some cases, it is recommended that this laser be used with $38 \%$ silver ammonium solution. These treatments should be performed under local anesthesia. [13] Because laser treatment had advantages with respect to control of hemorrhage and sterilization, laser is a valuable aid in direct pulp capping indeciduous and young permanenthuman teeth. [23] Melcer in 1987 first described laser treatment of exposed pulp tissues using the $\mathrm{CO} 2$ laser in dogs to achieve haemostasis and showed that $\mathrm{CO} 2$ laser produced new mineralized dentin without cellular modification of pulp tissue when tooth cavities were irradiated in beagles and primates. Ebihara used the $\mathrm{Nd}$ :YAG laser for exposed pulps of rats [23].

Pulpotomy by laser therapy was one of the most highly anticipated laser treatments because this treatment appeared to offer amputation of the pulp tissue at a satisfactory level. However, control of hemorrhage and amputation of pulp tissue without producing pulp damage was not always easy in narrow root canals. [13] The first laser pulpotomy was performed by Shoji et al. in 1985; 
they used $\mathrm{CO} 2$ laser in focused and defocused mode, with power levels of 3, 10, 30 and $60 \mathrm{~W}$, in dental pulp of dogs. They noticed coagulation necrosis and degeneration of the odontoblastic cell layer, without damaging the radicular pulp tissues. [24] The CO2 laser usually is used at a power of 1 to $4 \mathrm{~W}$. The laser irradiation should be conducted as intermittently as possible to prevent excessive exposure of laser energy. When it is necessary to ablate the pulp tissue into apical portion of the root canal, several laser exposures are required. As a result, the carbonization layer formed on the surface of the pulp tissue by the laser energy must be removed by irrigating alternatively with $3 \%$ hydrogen peroxide and $5.25 \%$ sodium hypochlorite. There are some problems concerning the application of the pulsed Nd:YAG laser for vital pulp amputation. Laser heat damage to the pulp tissue was the primary reason for the low success rate. It is recommended that the pulsed Nd:YAG laser should be used only for pulpal hemostasis, sedation, anti-inflammatory effects, and stimulation of the remaining pulpal cells. The $\mathrm{HeNe}$ and low-power semiconductor diode lasers and the middle-power semiconductor diode lasers are alternative lasers for these purposes. The middle-power semiconductor diode laser is being developed. [13] White noticed that the use of an $\mathrm{Nd}$ :YAG laser with the parameters $1 \mathrm{~W}$ power, $10 \mathrm{~Hz}$ repetition rate, total exposure time of $10 \mathrm{~s}$, does not significantly increase the temperature of the pulp tissue; these parameters represent the upper limit of laser application on pulp tissue [26].

Er,Cr:YSGG laser if applied for cutting enamel and dentin at $5 \mathrm{~W}$ and $6 \mathrm{~Hz}$ under water spray, access cavity preparation and enlargement of root canal orifices can be performed easily. Detailed examination must be done to prevent perforation into the periodontium and ledge formation on the root canal wall. [3] Mazeki K studied the efficacy of Er:YAG for root canal orifices on extracted teeth and in clinical cases and concluded thatLaser irradiation at $250 \mathrm{~mJ} /$ pulse and $8 \mathrm{~Hz}$ for approximately 60 sec in single root canal teeth and $120 \mathrm{sec}$ in multiple root canal teeth was adequate to clearly expose and enlarge root canal orifices, which were successfully prepared in 31 of 36 teeth $(86 \%)$ in the in vitro study. Walls enlarged by laser irradiation were slightly rough, but clean and almost no smear layer or debris was observed under SEM, in contrast to results obtained using Pieso reamers.

Laser irradiation at $160 \mathrm{~mJ} /$ pulse and $8 \mathrm{~Hz}$ for $60 \mathrm{sec}$ in anterior teeth, $90 \mathrm{sec}$ in premolar teeth, and $120 \mathrm{sec}$ in molar teeth was adequate to expose and enlarge root canal orifices clearly, which were successfully prepared in 10 of 11 teeth $(91 \%)$ in the clinical study. These results suggest that the preparation of root canal orifices by Er:YAG laser irradiation is useful in most cases, if appropriate parameters are selected. [27] Takeda F.H. observed the morphological changes on root canal walls after instrumentation and irrigation, and assessed the efficacy of conventional cleansing procedures and the effectiveness of Er:YAG laser in removing debris and smear layer from the root canal walls. The root canal walls irradiated by Er: YAG laser was free of debris, with an evaporated smear layer and open dentinal tubules. These results suggested that Er: YAG laser irradiation had an efficient cleaning effect on the prepared root canal walls. [28] Stabholz and colleague reported the development of new endodontic tip that can be used with an Er:YAG laser system. The beam of Er:YAG is delivered through a hollow tube, making it possible to develop an endodontic tip that allows lateral emission through a single opening at its far end. This newly designed side firing spiral tip was designed to fit the shape and volume of the root canal prepared by $\mathrm{Ni}-\mathrm{Ti}$ rotary instrumentation. It emit the Er:YAG laser irradiation laterally to the walls of root canal through the spiral slit located all along the tip. The tip is sealed at its far end, preventing the transmission of irradiation to and through the apical foramen of the tooth [24].

\section{Conclusion}

The clinical applications of any treatment modalities are given at a better quality after complete understanding of the mechanics of dental materials used. It's important for dentists to shift to quantum phase for qualitative treatments only if lasers provide us with value for time and life.

\section{References}

[1] Miserendino and Pick L and RM, Lasers in Dentistry, Quintessence publications, Chicago 1995, 341.

[2] Sulewski JG, "Historical survey of laser dentistry", Dental Clinics of North America, 44 (4), 717-751. 2002.

[3] Tranaeus S, Shi X-Q and Mansson BA, "Caries risk assessment: methods available to clinicians for caries detection", Community Dental Oral Epidemiology, 33, 265-273. 2005.

[4] Matsumoto K, "Lasers in endodontics", Dental Clinics of North America, 44, 889-906. 2000.

[5] Featherstone JDB, "Caries detection and prevention with laser energy" Dental Clinics of North America, 44(4), 955-969. 2000.

[6] Barnes MC, "Dental Hygiene Participation in Managing Incipient and Hidden Caries" Dental Clinics of North America, 49, 795-813. 2005.

[7] Stookey KG, "Quantitative Light Fluorescence: A technology for early monitoring of the caries process" Dental Clinics of North America, 49, 753-770. 2005.

[8] Krause F, Jepsen S and Braun A, "Comparison of two laser fluorescence devices for the detection of occlusal caries in vivo" European Journal of Oral Science, 115, 252-256. 2007.

[9] Jafarzadeh H, "Laser Doppler flowmetry in endodontics: A review” International Endodontic Journal, 42, 476-490.2009.

[10] Kimura Y, Smith PW and Matsumoto K, "Lasers in endodontics: a review” International Endodontic Journal, 33, 173-185.2000.

[11] Roebuck EM, Evans DJP, Stirrups D and Strang R, "The effect of wavelength, bandwidth, and probe design and position on assessing the vitality of anterior teeth with laser Doppler flowmetry" International Journal of Paeditric Denistry, 10, 213 220. 2000.

[12] Odor TM, Pitt FTR and McDonald F, "Effect of wavelength and bandwidth on the clinical reliability of laser Doppler recordings" Endodontics and Dental Traumatology, 12(1), 9-15.1996.

[13] Parker S, "Low level laser use in dentistry" British Dental Journal, 202, 131-138. 2007.

[14] Sasano T, Nakajima I, Shoji N, Kuriwada S, Sanjo D, Ogino H and Miyahara T, "Possible application of transmitted laser light for the assessment of human pulpal vitality" Endodontics and Dental Traumatology,13(2), 88-91. 1997.

[15] Parker S, "Introduction, history of lasers and laser light production" British Dental Journal, 202(1), 21-31. 2007.

[16] Moritz A, Schoop U, Strabl M and Wintner E, Cavity preparation Chapter 3 in: A Mortiz oral laser application, Quintessenz Verlags-GmbH, 2006, 75-138.

[17] Stabholz A, Neev J, Liaw LH, Stabholz A, Khayat and Torabinejad M, "Sealing of human dentinal tubules by $\mathrm{XeCl} 308$ $\mathrm{nm}$ excimer laser" Journal of Endodontics, 19(6), 267-71, 1993.

[18] Walsh LJ, "The current status of laser applications in dentistry", Australian Dental Journal, 48(3), 146-155. 2003. 
[19] Powell GL, Morton TH and Whisenant BK, "Argon laser oral safety parameters for teeth", Lasers Surg Med, 13, 548-552. 1993.

[20] Anic I, Pavelic B and Matsumoto K, "In vitro pulp chamber temperature rises associated with the argon laser polymerization of composite resin", Lasers Surg Med, 19, 438-444. 1996.

[21] Westerman GH, Hicks MJ and Flaitz CM, "Surface morphology of sound enamel after Argon laser irradiation: An in vitro scanning electron microscopic study", J Clin Pediat Dent, 21, 55-59. 1996.

[22] Lin CP, Lee BS, Lin FH, Kok SH and Lan WH, "Phase, compositional, and morphological changes of human dentin after Nd:YAG laser treatment", Journal of Endodontics, 27(6), 389-93. 2001.

[23] Moritz A, Schoop U, Goharkhay K and Sperr W, "The CO2 laser as an aid in direct pulp capping" Journal of Endodontics, 24, 248251. 1998.
[24] Stabholz A, Sahar-Helft S and Moshonov J, "Lasers in endodontics" Dental Clinics of North America, 48, 809-832. 2004.

[25] Moritz A, Schoop U, Goharkhay K and Sperr W, “Advantages of a pulsed $\mathrm{CO} 2$ laser in direct pulp capping: a long term in vivo study", Lasers in Surgery and Medicine, 22, 288-293. 1998.

[26] White JM, Fagan MC and Goodis HE, "Intrapulpal temperature during pulsed Nd:YAG laser treatment of dentin, in vitro", Journal of Periodontology, 65, 255-259. 1994.

[27] Mazeki K, Kimura Y, Yokoyama K and Matsumoto K, "Preparation of Root Canal Orifices by Er:YAG Laser Irradiation: In Vitro and Clinical Observations" Journal of Clinical Laser in Medcine and Surgery, 21(2), 85-91. 2002.

[28] Takeda FH, Harashima T, Eto JN, Kimura Y and Matsumoto K, "Effect of Er:YAG laser treatment on the root canal walls of human teeth”, Endodotic Dental Traumatology, 14, 270-273.1998. 